\title{
ANTIOXIDANT CAPACITY AND QUANTIFICATION ANALYSIS OF CHEMICAL CONSTITUENTS ISOLATED FROM TETRASTIGMA HEMSLEYANUM LEAVES
}

\author{
CHONG-LU SUN ${ }^{1 \#}$, SHI-MIN LI ${ }^{1 \#,}$, SONG-LIN YU ${ }^{1}$, YING-XIN YANG ${ }^{1}$, YU-JIONG ZHANG ${ }^{1}$, \\ SU-FANG HAN ${ }^{3}$, and XIN PENG ${ }^{1,2 *}$
}

${ }^{1}$ Zhejiang Pharmaceutical College, Ningbo 315100, PR China

${ }^{2}$ Ningbo Research Institute of Zhejiang University, Ningbo 315100, PR China

${ }^{3}$ Zhejiang Academy of Forestry, Hangzhou 310023, PR China

\begin{abstract}
Tetrastigma hemsleyanum is an edible and medicinal plant in China. There are little researches on its leaves as the root is the conventionally used part. In this study, chemical constituents of $T$. hemsleyanum leaves (THL) were isolated and their antioxidant activities were studied. A total of fifteen flavonoids were obtained from this plant. They were kaempferol-3-O- $\beta$-rutinoside (1), quercetin (2), astragalin (3), quercitrin (4), isoquercitrin (5), rutin (6), vitexin (7), isovitexin (8), orientin (9), isoorientin (10), vicenin-2 (11), lucenin-2 (12), lucenin-1 (13), vicenin-1 (14), and vicenin-3 (15). Among them, compounds 12-15 were isolated from $T$. hemsleyanum for the first time. The results of antioxidant assays revealed that compounds 2, 4-6, 9-14 possess encouraging antioxidant activities. In addition to the antioxidant activity study, a sensitive and rapid analytical method was developed in this study to determine these antioxidants simultaneously by using ultra-performance liquid chromatography coupled with triple-quadrupole tandem mass spectrometry (UPLC-MS/MS). In conclusion, THL could be a new source for functional foods and a potential candidate for antioxidant agents.
\end{abstract}

Keywords: Tetrastigma hemsleyanum, antioxidant activity, chemical constituents, UPLC-MS/MS

Tetrastigma hemsleyanum Diels et Gilg (T. hemsleyanum) belongs to the family of Vitaceae and is a well-known edible and medicinal plant in China. It grows mainly in the area of the Yangtze River including Zhejiang, Fujian, Jiangxi, Guangxi, and Yunnan provinces in China (1). Previous pharmacology studies showed that the bulbous roots of T. hemsleyanum had multiple functions, such as antiinflammation, anti-virus, and anti-tumor activities, and it can relieve cold and rheumatic pain, etc (2-4). The extracts of bulbous roots have been used as therapeutic agents in the Chinese herbal market. So far, most of the researches focused on the chemical composition and biological activities of the roots. But the phytochemical composition of $T$. hemsleyanum leaves (THL) is largely unknown, and little information is available on their healthful functions (5). However, extracts of the THL have been used for the treatment of hepatitis, rheumatoid arthritis, viral meningitis, and other diseases in folk medicine for a long time $(6,7)$. The results of our preliminary experiment indicated that the n-butanol soluble fraction of THL might contain some antioxidants due to its significant free radical scavenging activity. Therefore, it is necessary to provide scientific evidence for the characterization of antioxidant substances in THL.

Many chronic diseases, including cardiovascular diseases, chronic inflammation, cancer, and Alzheimer's disease, are associated with the harmful effects induced by excessive reactive oxygen species (ROS) and reactive nitrogen species (RNS). Some antioxidants may play a dominant role in the prevention of neurodegenerative diseases by scavenging free radicals and by relieving the oxidation of biomolecules $(8,9)$. In order to reduce the harm of reactive oxygen free radicals, more and more antioxidants have been explored, such as vitamin $\mathrm{C}$, vitamin $\mathrm{E}$, butylated hydroxyanisole (BHA), etc. With the enhancement of awareness of potential adverse effects, the use of these chemosynthetic antioxidants was limited by legislation (10). Compared with chemosynthetic antioxidants, natural antioxidants have the advantages of low toxicity and

\footnotetext{
\# These authors contributed equally to this work

* Corresponding author: e-mail: pengx@ nit.zju.edu.cn
} 
high absorbance (11). Therefore, it seems to be a feasible approach to look for safe and effective antioxidant ingredients from traditional Chinese herbs (12).

In this study, 15 compounds were isolated and identified from THL. Then, their antioxidant activities were determined and ten of them were found to have strong antioxidant activities. In addition, an analytical method for simultaneous determination of the ten antioxidants was established by using ultra-performance liquid chromatography coupled with triple-quadrupole tandem mass spectrometry (UPLC-MS/MS) for the first time.

\section{EXPERIMENTAL}

\section{Chemicals and reagents}

1,1-Diphenyl-2-picrylhydrazyl and 2,4,6-tripyridyl triazine were purchased from Beijing Solarbio Technology Co., Ltd. Acetonitrile was of HPLC grade (Tedia, USA), and formic acid was of HPLC grade (Merck, Germany). Deionized water $\left(\mathrm{H}_{2} \mathrm{O}\right)$ was purified by a super purification system (Eped Technology Development, Nanjing, China). Other reagent solutions were of analytical grades, such as ethanol, $\mathrm{CHCl}_{3}, \mathrm{MeOH}$, and EtOAc, and were purchased from Sinopharm Chemical Reagent Co., Ltd.

\section{General experimental procedures}

NMR spectra were determined using a Bruker spectrometer at $400 \mathrm{MHz}$ for ${ }^{1} \mathrm{H}$ and $100 \mathrm{Mz}$ for ${ }^{13} \mathrm{C}$ in DMSO as solvent using tetramethylsilane (TMS) as internal standard. HERSIMS spectra were measured using a Bruker MaXis ultra-high-resolution TOF apparatus with electrospray ionization (ESI). UV was recorded by a JASCO V-650 spectrophotometer. Prep-HPLC separation was performed on the P3500 instrument (Dalian, China) with Shimadzu column $\mathrm{C}_{18}(250 \times 10 \mathrm{~cm}, 5.0 \mu \mathrm{m})$. Pre-TLC was carried out on precoated silica gel F254 plates $(20 \times$ $20 \mathrm{~cm}, 1.0 \mathrm{~mm}$ layer thickness, Merck). Sephadex LH-20 (25-100 $\mu \mathrm{m}$, Solarbio, China) and silica gel (200-300 mesh, Qingdao Haiyang) were used for column chromatography. TLC was performed on precoated $0.25 \mathrm{~mm}$ thick Merck (Darmstadt, Germany) silica gel F254 aluminum-backed plates. Fractions were monitored by TLC with visualization under UV light (254 and $365 \mathrm{~nm}$ ) and visualized by spraying with $5 \% \mathrm{H}_{2} \mathrm{SO}_{4}$-ethanol reagent and heating at $120^{\circ} \mathrm{C}$ for $3 \mathrm{~min}$. The quantification analysis was performed using the LC-20AD system (Shimadzu, Japan) coupled with SCIEX QTRAP 4500 ESI-MS/MS (SCIEX, USA) consisting of a triple quadrupole and ion source of electrospray ionization (ESI). All other chemicals and solvents were of analytical grade.

\section{Plant material}

The fresh T. hemsleyanum leaves were collected at a different time from nine provinces, including Zhejiang, Anhui, Guangxi, Guangdong, Jiangxi, Chongqing, Fujian, Guizhou, and Yunnan.

Table 1. The sample information of $T$. hemsleyanum from different habitats.

\begin{tabular}{|c|c|c|c|c|}
\hline Accession No. & Gathering areas & Latitude & Longitude & Collection time \\
\hline S-1 & Wuyi Zhejiang & $\mathrm{N} 28^{\circ} 76^{\prime}$ & $\mathrm{E} 119^{\circ} 41^{\prime}$ & 2019.10 \\
\hline $\mathrm{S}-2$ & Suichang Zhejiang & $\mathrm{N} 29^{\circ} 31^{\prime}$ & E118 $58^{\prime}$ & 2019.10 \\
\hline $\mathrm{S}-3$ & Lishui Zhejiang & $\mathrm{N} 28^{\circ} 26^{\prime}$ & $\mathrm{E} 119^{\circ} 50^{\prime}$ & 2019.10 \\
\hline S-4 & Longquan Zhejiang & $\mathrm{N} 28^{\circ} 08^{\prime}$ & $\mathrm{E} 119^{\circ} 13^{\prime}$ & 2019.10 \\
\hline $\mathrm{S}-5$ & Quzhou Zhejiang & $\mathrm{N} 28^{\circ} 66^{\prime}$ & $\mathrm{E} 118^{\circ} 91^{\prime}$ & 2019.11 \\
\hline S-6 & Yongkang Zhejiang & $\mathrm{N} 28^{\circ} 45^{\prime}$ & E119 $53^{\prime}$ & 2019.10 \\
\hline $\mathrm{S}-7$ & Ningbo Zhejiang & $\mathrm{N} 29^{\circ} 46^{\prime}$ & $\mathrm{E} 121^{\circ} 31^{\prime}$ & 2018.11 \\
\hline $\mathrm{S}-8$ & Chongming Guangxi & $\mathrm{N} 22^{\circ} 45^{\prime}$ & $\mathrm{E} 107^{\circ} 21^{\prime}$ & 2018.10 \\
\hline S-9 & Guilin Guangxi & $\mathrm{N} 24^{\circ} 78^{\prime}$ & $\mathrm{E} 110^{\circ} 87^{\prime}$ & 2019.10 \\
\hline S-10 & Baile Guangxi & $\mathrm{N} 24^{\circ} 79^{\prime}$ & E1145의 & 2018.10 \\
\hline $\mathrm{S}-11$ & Huangshan Anhui & $\mathrm{N} 30^{\circ} 13^{\prime}$ & $\mathrm{E} 118^{\circ} 18^{\prime}$ & 2019.10 \\
\hline $\mathrm{S}-12$ & Qingyuan Guangdong & $\mathrm{N} 23^{\circ} 81^{\prime}$ & $\mathrm{E} 115^{\circ} 78^{\prime}$ & 2019.01 \\
\hline S-13 & Shangrao Jiangxi & $\mathrm{N} 28^{\circ} 67^{\prime}$ & $\mathrm{E} 117^{\circ} 34^{\prime}$ & 2018.11 \\
\hline S-14 & Sanhe Chongqing & $\mathrm{N} 29^{\circ} 35^{\prime}$ & $\mathrm{E} 106^{\circ} 11^{\prime}$ & 2018.11 \\
\hline $\mathrm{S}-15$ & Chenggong Yunnan & $\mathrm{N} 24^{\circ} 51^{\prime}$ & $\mathrm{E} 102^{\circ} 50^{\prime}$ & 2019.10 \\
\hline S-16 & Sanming Fujian & $\mathrm{N} 26^{\circ} 12^{\prime}$ & $\mathrm{E} 117^{\circ} 31^{\prime}$ & 2019.10 \\
\hline S-17 & Xinyi Guizhou & $\mathrm{N} 24^{\circ} 59^{\prime}$ & $\mathrm{E} 104^{\circ} 50^{\prime}$ & 2019.11 \\
\hline
\end{tabular}


The detailed sample information was shown in Table 1. The specimens were identified and authenticated by Prof. Xin Peng, Zhejiang University. All voucher specimens were deposited at the Biotechnology Institute of Zhejiang Pharmaceutical College, Zhejiang province in China.

\section{Extraction and isolation}

T. hemsleyanum sample (S-7) was provided by Shengwang Biotechnology Corporation, and used for the extraction and separation of compounds in subsequent studies. The air-dried and powdered plant of THL (5000 g) was extracted with $20 \mathrm{~L}$ of $70 \%$ ethanol at room temperature for $3 \mathrm{~h}$, repeat 3 times. The extract was filtered and evaporated to dryness under vacuum at $45^{\circ} \mathrm{C}$. The obtained dry extract $(800 \mathrm{~g})$ was then suspended in hot $\mathrm{MeOH}-\mathrm{H}_{2} \mathrm{O}(1: 10, \mathrm{v} / \mathrm{v})$ and successively partitioned with n-hexane, methylene chloride, ethyl acetate, n-butanol to yield the corresponding fractions. Each fraction was tested for the radical-scavenging effect by 1,1-diphenyl2-picrylhydrazyl (DPPH) assay. Among these fractions, the n-butanol soluble fraction $(150 \mathrm{~g})$ showed the most significant free radical scavenging activity. The n-butanol soluble fraction was subjected to chromatography on a Sephadex LH-20 column (MeOH) to afford six fractions (Fr.1-Fr.6). Fr.1 was separated on silica gel column chromatography (EtOAc$\mathrm{MeOH}, 5:$ 1) to give three subfractions (Subfr.1.1Subfr.1.3). Fr.2 was subjected to silica gel column chromatography $\left(\mathrm{CHCl}_{3} / \mathrm{MeOH} 10: 1\right)$ to yield compound 1 (21 mg) and compound 6 (22 $\mathrm{mg})$, respectively. Compound 2 (18 mg) and compound 5 (22 mg) were crystallized from Fr.3 and Fr.4, respectively. Fr.5 was subjected to silica gel column chromatography (EtOAc/EtOH/ $\mathrm{H}_{2} \mathrm{O}$ 10:1:0.2) and prepHPLC $\left(\mathrm{ACN} / \mathrm{H}_{2} \mathrm{O}\right)$ to obtain compound $4(27 \mathrm{mg})$, compound $7(30 \mathrm{mg})$, and compound $8(29 \mathrm{mg})$. Fr.6 was purified by prep-TLC (EtOH/MeOH 2:1) and prep-HPLC $\left(\mathrm{ACN} / \mathrm{H}_{2} \mathrm{O}\right)$ to obtain compound 3 (27 mg), compound 9 (29 mg), and compound 10 (38 mg). Subfr.1.1 was purified by prep-HPLC using Shimadzu column $\left(\mathrm{ACN} / \mathrm{H}_{2} \mathrm{O}\right)$ to give compound 12 (63 mg). Subfr.1.2 was crystallized with $\mathrm{MeOH}$ to yield compound 13 (88 mg). Subfr.1.3 was purified by prep-TLC (EtOH/MeOH 1: 1) and prep-HPLC (ACN/ $\mathrm{H}_{2} \mathrm{O}$ ) to obtain compound 11 (53 mg), compound 14 (69 mg), and compound 15 (86 mg).

\section{Evaluation of antioxidant activity DPPH free radical scavenging activity assay}

The evaluation of DPPH free radical scavenging activity was carried out according to the previously reported method with some modifications
(13). $1.0 \mathrm{~mL}$ of methanol solutions at various concentrations $(1-100 \mu \mathrm{g} / \mathrm{L})$ was mixed with $2.0 \mathrm{~mL}$ of DPPH in ethanol $(0.1 \mathrm{mM})$. The reaction mixture was shaken and incubated at room temperature. The absorbance of the reaction mixture was measured at $517 \mathrm{~nm}$ by UV-Vis spectrophotometer $30 \mathrm{~min}$ later. The inhibition percentage of DPPH free radicals was calculated as follows: inhibition $\%=\left[\left(\mathrm{A}_{\text {blank }}-\mathrm{A}_{\text {sample }}\right) /\right.$ $\left.\mathrm{A}_{\text {blank }}\right] \times 100$; $\mathrm{A}_{\text {blank }}$ was the absorbance of DPPH without sample, and $\mathrm{A}_{\text {sample }}$ was the absorbance of DPPH with a sample in it. The results were expressed by $\mathrm{IC}_{50}$, which represented the sample concentration required to inhibit $50 \% \mathrm{DPPH}$ free radicals. $\mathrm{IC}_{50}$ was measured by the relationship curve between concentration and absorbance. The results presented are the mean of two experiments.

\section{Scavenging ABTS free radical activity assay}

2,2'-azino-bis(3-ethylbenzothiazoline-6-sulfonic acid) (ABTS) free radical scavenging activity was measured according to the previously reported method with some modifications (14). $7 \mathrm{mM}$ ABTS solution prepared in water was mixed with $2.45 \mathrm{mM}$ potassium persulphate solution and the mixture was kept at room temperature in dark for $12-16 \mathrm{~h}$ to produce $\mathrm{ABTS}^{+}$free radicals. The absorbance of the $\mathrm{ABTS}^{+}$solution was set at $0.700 \pm 0.02$ at $734 \mathrm{~nm}$ by diluting with ethanol. Then, $2 \mathrm{~mL}$ of $\mathrm{ABTS}^{+}$solution was added to $1.0 \mathrm{~mL}$ of each sample solution at various concentrations (1-100 $\mu \mathrm{g} / \mathrm{L})$. The mixture was incubated for $4 \mathrm{~min}$ and the absorbance was measured at $734 \mathrm{~nm}$. The antioxidant activity was calculated by the same equation as the DPPH method.

\section{Ferric-reducing antioxidant power (FRAP) assay}

FRAP assay was carried out using the method based on a previous study (15). The capability of reducing the tripyridyltriazine complex of $\mathrm{Fe}^{3+}$ i.e. $\left(\mathrm{Fe}^{3+} / \mathrm{TPTZ}\right)$ into blue-colored complex $\mathrm{Fe}^{2+}$ / TPTZ was measured. 0.3 $\mathrm{M}$ acetate buffer ( $\mathrm{pH} 3.6)$, $10 \mathrm{mmol}$ of TPTZ solution in $40 \mathrm{mmol}$ of $\mathrm{HCl}$, and $20 \mathrm{mmol}$ of ferric chloride hexahydrate solutions in the ratio of 10: 1: $1(\mathrm{v} / \mathrm{v} / \mathrm{v})$ were mixed and heated at $37^{\circ} \mathrm{C}$ to prepare FRAP reagent. A series of standard solutions of ferrous ions $(0.2,0.4,0.6,0.8,1.0$, and $1.2 \mathrm{mmol} / \mathrm{L})$ were prepared by diluting the stock solution $(10 \mathrm{mmol} / \mathrm{L})$ with water for the establishment of standard curves. $0.2 \mathrm{~mL}$ of the above standard solution was added into a $3 \mathrm{~mL}$ FRAP reagent. The reaction mixture was incubated at room temperature for $15 \mathrm{~min}$ and the absorbance was measured at $595 \mathrm{~nm}$. Antioxidant activity was calculated according to the standard curve (for example, the absorbance of $0.2 \mathrm{mmol} / \mathrm{L}$ sample is equivalent to 
$1 \mathrm{mmol} / \mathrm{L} \mathrm{FeSO}_{4}$ standard solution, the relative antioxidant ability of the sample is 5).

\section{UPLC conditions}

UPLC analysis was performed on a Shimadzu LC-20 AD system (Shimadzu Crop., Tokyo, Japan) equipped with an autosampler, a binary solvent delivery pump, and Analyst Software 1.6.3. The separation was performed on the Kinetex XB- $\mathrm{C}_{18}$ column (50 mm $\times 2.1 \mathrm{~mm}, 2.6 \mu \mathrm{m}$ ), with the mobile phase consisting of aqueous formic acid ( $0.1 \%$ in water, solvent $\mathrm{A})$ and acetonitrile (solvent B) in gradient elution mode. The gradient for the negative electrospray ionization (ESI) mode was started and held at $10 \% \mathrm{~B}$ for $1 \mathrm{~min}$, raised linearly from 10 to $60 \% \mathrm{~B}$ during the next $6 \mathrm{~min}$, and then maintained at $60 \% \mathrm{~B}$ for $2 \mathrm{~min}$. Then, the mobile phase returned to $10 \% \mathrm{~B}$ within $2 \mathrm{~min}$ and the system was equilibrated for $5 \mathrm{~min}$ before the next run. The flow rate was $0.1 \mathrm{~mL} / \mathrm{min}$, the injection volume was $1.0 \mu \mathrm{L}$ and the column temperature was $20^{\circ} \mathrm{C}$.

\section{Mass Spectrometric Conditions}

An AB-SCIEX 4500 Triple-Quad mass spectrometer was connected to the Shimadzu LC-20 AD Ultra Performance Liquid Chromatograph via ESI interface for analysis. The MultiQuant software packages were used for data acquisition and processing. The determination for all analytes was carried out on tandem mass spectrometry in the negative ion ESI mode and multiple reaction monitoring (MRM) mode. The parameters in the negative ion mode were as follows: ion source temperature, $550^{\circ} \mathrm{C}$; ion spray voltage, $4.5 \mathrm{kV}$; air curtain gas, 40 psi; spray gas, $50 \mathrm{psi}$; auxiliary heating gas, 50 psi. The collision gas $\left(\mathrm{N}_{2}\right)$ flow was set at medium.

\section{Preparation of standard solutions}

A mixed standard stock solution containing compounds 2, 4-6, 9-14 was prepared in methanol. Then it was diluted with methanol to obtain a series concentrations of working standard solutions within ranges: compound 2, 5-500 ng/mL; compound 4, $5-500 \mathrm{ng} / \mathrm{mL}$; compound 5, 5-500 ng/mL; compound 6, 5-1000 ng/mL; compound 9, 5-1000 ng/mL; compound $10,5-2000 \mathrm{ng} / \mathrm{mL}$; compound $11,5-2000 \mathrm{ng} /$ $\mathrm{mL}$; compound $12,5-2000 \mathrm{ng} / \mathrm{mL}$; compound 13 , $5-1000 \mathrm{ng} / \mathrm{mL}$; compound 14, 5-2000 ng/mL. All solutions were stored at $-20^{\circ} \mathrm{C}$ and filtered through a $0.22 \mu \mathrm{m}$ membrane prior to injection.

\section{Preparation of sample solutions}

Seventeen batches of $300 \mathrm{mg}$ dried and powdered THL were soaked with $50 \mathrm{~mL}$ of $75 \%$ methanol for 30 minutes, then ultrasonicated twice for
30 minutes, and finally made up to a volume of $50 \mathrm{~mL}$ with $75 \%$ methanol. The solution was filtered through a $0.22 \mu \mathrm{m}$ membrane and the filtrates were diluted ten-fold with methanol. Then $1.0 \mu \mathrm{L}$ of aliquot was injected into the UPLC-MS/MS system for analysis. All the experiments were repeated three times.

\section{Method validation}

Various concentrations of standard solutions were prepared for the establishment of calibration curves. It was obtained by linear regression between the peak area $(\mathrm{Y})$ and analyte concentration $(\mathrm{X})$, the corresponding linear range and correlation coefficient were also gained. The limits of detection (LOD) and limits of quantification (LOQ) were determined when the signal-to-noise ratio was 10 and 3 , respectively. They were determined by serial dilution of standard solution using the above-mentioned UPLC-MS/MS conditions. The repeatability, intraday, and inter-day precisions were expressed as percentage relative standard deviations (RSD). The mixture standard solution was measured six times during a single day and six consecutive days for evaluation for intra-day and inter-day based on changes in the peak area. Repeatability was confirmed with six independent samples from the same batch and the variations were expressed by RSD. Recovery was used to evaluate the accuracy of this method. It was determined by comparing the average peak areas of extracted samples at low, medium, and high concentrations with the average peak areas of spike-afterextraction samples.

\section{RESULTS AND DISCUSSION}

\section{Identification of compounds}

The n-butanol fraction was subjected to separation by various sessions of chromatography to produce 15 compounds (Figure 1). Based on the analysis of chemical property and spectral data, compounds 1-15 were identified as: kaempferol-3-O- $\beta$-rutinoside (1); quercetin (2); astragalin (3); quercitrin (4); isoquercitrin (5); rutin (6); vitexin (7); isovitexin (8); orientin (9); isoorientin (10); vicenin-2 (11); lucenin-2 (12); lucenin-1 (13); vicenin-1 (14); vicenin-3(15), respectively. Among them, compounds 12-15 were isolated from $T$. hemsleyanum for the first time.

Kaempferol-3-O- $\beta$-rutinoside (1): ESI-MS $\mathrm{m} / \mathrm{z}: 595[\mathrm{M}+\mathrm{H}]^{+} .{ }^{1} \mathrm{H}-\mathrm{NMR}\left(400 \mathrm{MHz}, \mathrm{DMSO}-\mathrm{d}_{6}\right.$ ) $\delta: 12.55(1 \mathrm{H}, \mathrm{s}, 5-\mathrm{OH}), 10.85(1 \mathrm{H}, \mathrm{s}, 7-\mathrm{OH}), 10.13$ $\left(1 \mathrm{H}, \mathrm{s}, 4^{\prime}-\mathrm{OH}\right), 7.97\left(2 \mathrm{H}, \mathrm{d}, J=8.8 \mathrm{~Hz}, \mathrm{H}-2^{\prime}, 6^{\prime}\right), 6.86$ $\left(2 \mathrm{H}, \mathrm{d}, J=8.8 \mathrm{~Hz}, \mathrm{H}-3^{\prime}, 5^{\prime}\right), 6.40(1 \mathrm{H}, \mathrm{d}, J=1.6 \mathrm{~Hz}$, H-8), $6.20(1 \mathrm{H}, \mathrm{d}, J=1.6 \mathrm{~Hz}, \mathrm{H}-6), 5.32(1 \mathrm{H}, \mathrm{d}$, $J=8.0 \mathrm{~Hz}$, Glu-H-1), 4.38 (H, s, Rha-H-1), 0.98 
<smiles>O=c1c(-c2ccc(O)cc2)c(-c2ccc(O)cc2)oc2cc(O)cc(O)c12</smiles>

compound $1:$ R=Glu-Rha compound $3: \mathrm{R}=\mathrm{Glu}$<smiles>O=c1c(O)c(-c2ccc(O)c(O)c2)oc2cc(O)cc(O)c12</smiles>

Figure 1. Structures of compound 1-15.

(3H, d, $J=3.2 \mathrm{~Hz}$, Rha- $\left.\mathrm{CH}_{3}\right) ;{ }^{13} \mathrm{C}-\mathrm{NMR}(100 \mathrm{MHz}$, DMSO- $\left.\mathrm{d}_{6}, \delta_{c}\right)$ See Table 2 . These data were in consistent with those of kaempferol-3-O- $\beta$-rutinoside (16).

Quercetin (2): ESI-MS m/z: $303[\mathrm{M}+\mathrm{H}]^{+}$. ${ }^{1} \mathrm{H}-\mathrm{NMR}\left(400 \mathrm{MHz}, \mathrm{DMSO}-\mathrm{d}_{6}\right) \delta: 12.48(1 \mathrm{H}, \mathrm{s}$, 5-OH), 9.59 (1H, s, 7-OH), 9.32 (2H, s, 3', 4'-OH), $7.68\left(1 \mathrm{H}, \mathrm{d}, J=2.0 \mathrm{~Hz}, \mathrm{H}-2^{\prime}\right), 7.54(1 \mathrm{H}, \mathrm{dd}, J=8.8$, $\left.2.2 \mathrm{~Hz}, \mathrm{H}-6^{\prime}\right), 6.88\left(1 \mathrm{H}, \mathrm{d}, J=8.8 \mathrm{~Hz}, \mathrm{H}-5^{\prime}\right), 6.40(1 \mathrm{H}$, d, $J=2.0 \mathrm{~Hz}, \mathrm{H}-8), 6.18(1 \mathrm{H}, \mathrm{d}, J=2.0 \mathrm{~Hz}, \mathrm{H}-6)$; ${ }^{13} \mathrm{C}-\mathrm{NMR}\left(100 \mathrm{MHz}, \mathrm{DMSO}-\mathrm{d}_{6}, \delta_{\mathrm{c}}\right.$ ) See Table 2. These data were in consistent with those of quercetin (17).

Astragalin (3): ESI-MS m/z: 449[M+H $]^{+}$. ${ }^{1} \mathrm{H}-\mathrm{NMR}\left(400 \mathrm{MHz}, \mathrm{DMSO}-\mathrm{d}_{6}\right) \delta: 12.61(1 \mathrm{H}, \mathrm{s}$, 5-OH), $10.22(1 \mathrm{H}, \mathrm{s}, 7-\mathrm{OH}), 8.05(2 \mathrm{H}, \mathrm{d}, J=9.2 \mathrm{~Hz}$, $\left.\mathrm{H}-2^{\prime}, 6^{\prime}\right), 6.88\left(2 \mathrm{H}, \mathrm{d}, J=9.2 \mathrm{~Hz}, \mathrm{H}-3^{\prime}, 5^{\prime}\right), 6.43(1 \mathrm{H}$, $\mathrm{d}, J=2.0 \mathrm{~Hz}, \mathrm{H}-8), 6.21(1 \mathrm{H}, \mathrm{d}, J=2.1 \mathrm{~Hz}, \mathrm{H}-6)$, $5.45(1 \mathrm{H}, \mathrm{d}, J=7.2 \mathrm{~Hz}$, Glu-H-1), 2.90 3.57 (6H, $\mathrm{m}$, Glu-H); ${ }^{13} \mathrm{C}-\mathrm{NMR}\left(100 \mathrm{MHz}, \mathrm{DMSO}-\mathrm{d}_{6}, \delta_{c}\right)$ See Table 2. These data were in consistent with those of astragalin (18).<smiles>[2H]c1c(O)c([2H])c2oc(-c3ccc(O)cc3)cc(=O)c2c1O</smiles>

compound $7: \mathrm{R}_{1}=\mathrm{Glu}, \mathrm{R}_{2}=\mathrm{H}$ compound $8: \mathrm{R}_{1}=\mathrm{H}, \mathrm{R}_{2}=\mathrm{Glu}$ compound $11: R_{1}=G l u, R_{2}=G l u$ compound $14: R_{1}=G l u, R_{2}=X y l$ compound $15: R_{1}=X y l, R_{2}=$ Glu<smiles>[R]c1c(O)c([2H])c2oc(-c3ccc(O)c(O)c3)cc(=O)c2c1O</smiles>

Quercitrin (4): ESI-MS m/z: 449[M+H] $]^{+}$. ${ }^{1} \mathrm{H}-\mathrm{NMR}\left(400 \mathrm{MHz}, \mathrm{DMSO}-\mathrm{d}_{6}\right) \delta: 12.65(1 \mathrm{H}, \mathrm{s}$, OH-5), 10.86 (1H, s, OH-7), $9.69(1 \mathrm{H}, \mathrm{s}, \mathrm{OH}-$ 4'), $9.36\left(1 \mathrm{H}, \mathrm{s}, \mathrm{OH}-3^{\prime}\right), 7.30(1 \mathrm{H}, \mathrm{d}, J=2.0 \mathrm{~Hz}$, H-2'), 7.26 (1H, dd, $J=8.4,2.4 \mathrm{~Hz}, \mathrm{H}-6$ '), 6.86 $(1 \mathrm{H}, \mathrm{d}, J=8.4 \mathrm{~Hz}, \mathrm{H}-5$ '), $6.39(1 \mathrm{H}, \mathrm{d}, J=2.0 \mathrm{~Hz}$, H-8), $6.20(1 \mathrm{H}, \mathrm{d}, J=2.0 \mathrm{~Hz}, \mathrm{H}-6), 5.25(1 \mathrm{H}, \mathrm{d}$, $J=1.2 \mathrm{~Hz}$, Rha-H-1), $0.82(3 \mathrm{H}, \mathrm{d}, J=7.0 \mathrm{~Hz}$, Rha-H-6); ${ }^{13} \mathrm{C}-\mathrm{NMR}\left(100 \mathrm{MHz}, \mathrm{DMSO}-\mathrm{d}_{6}, \delta_{\mathrm{c}}\right.$ ) See Table 2. These data were in consistent with those of quercitrin (19).

Isoquercitrin (5): ESI-MS m/z: $465[\mathrm{M}+\mathrm{H}]^{+}$. ${ }^{1} \mathrm{H}-\mathrm{NMR}(400 \mathrm{MHz}, \mathrm{DMSO}-\mathrm{d} 6) \delta: 12.62(1 \mathrm{H}, \mathrm{s}$, 5-OH), $10.89(1 \mathrm{H}, \mathrm{s}, 7-\mathrm{OH}), 9.75\left(1 \mathrm{H}, \mathrm{s}, 4^{\prime}-\mathrm{OH}\right)$, $9.22\left(1 \mathrm{H}, \mathrm{s}, 3^{\prime}-\mathrm{OH}\right), 7.68\left(1 \mathrm{H}, \mathrm{d}, J=1.6 \mathrm{~Hz}, \mathrm{H}-2^{\prime}\right)$, $7.54\left(1 \mathrm{H}, \mathrm{dd}, J=2.0,8.5 \mathrm{~Hz}, \mathrm{H}-6^{\prime}\right), 6.82(1 \mathrm{H}, \mathrm{d}$, $J=8.4 \mathrm{~Hz}, \mathrm{H}-5$ '), 6.41 (1H, d, $J=2.0 \mathrm{~Hz}, \mathrm{H}-8), 6.20$ $(1 \mathrm{H}, \mathrm{d}, J=2.0 \mathrm{~Hz}, \mathrm{H}-6), 5.38(1 \mathrm{H}, \mathrm{d}, J=7.6 \mathrm{~Hz}$, Glu-H-1); ${ }^{13} \mathrm{C}-\mathrm{NMR}\left(100 \mathrm{MHz}, \mathrm{DMSO}-\mathrm{d}_{6}, \delta_{c}\right)$ See Table 2. These data were in consistent with those of isoquercitrin (20-21). 
Table 2. NMR Spectroscopic data of compound 1-15.

\begin{tabular}{|c|c|c|c|c|c|c|c|c|c|c|c|c|c|c|c|}
\hline Compound & 1 & 2 & 3 & 4 & 5 & 6 & 7 & 8 & 9 & 10 & 11 & 12 & 13 & 14 & 15 \\
\hline position & $\delta_{c}$ & $\delta_{c}$ & $\delta_{c}$ & $\delta_{c}$ & $\delta_{\mathrm{c}}$ & $\delta_{c}$ & $\delta_{c}$ & $\delta_{c}$ & $\delta_{c}$ & $\delta_{c}$ & $\delta_{c}$ & $\delta_{\mathrm{c}}$ & $\delta_{c}$ & $\delta_{c}$ & $\delta_{c}$ \\
\hline 2 & 156.5 & 146.8 & 156.4 & 156.5 & 156.3 & 156.7 & 164.0 & 163.5 & 164.1 & 163.6 & 164.2 & 164.3 & 164.3 & 164.2 & 164.0 \\
\hline 3 & 133.2 & 135.7 & 133.2 & 134.2 & 133.6 & 133.6 & 102.5 & 102.8 & 102.4 & 102.8 & 102.5 & 102.8 & 102.5 & 102.5 & 102.2 \\
\hline 4 & 177.4 & 175.8 & 177.5 & 177.8 & 177.5 & 177.4 & 182.1 & 181.9 & 182.0 & 181.8 & 182.4 & 182.3 & 182.3 & 182.4 & 182.4 \\
\hline 5 & 161.2 & 156.1 & 161.2 & 161.3 & 161.3 & 161.3 & 160.4 & 161.2 & 160.4 & 160.6 & 158.7 & 158.6 & 158.3 & 158.3 & 158.2 \\
\hline 6 & 93.7 & 93.3 & 98.7 & 98.7 & 98.7 & 98.7 & 98.2 & 108.9 & 98.2 & 108.8 & 107.6 & 107.5 & 108.1 & 108.2 & 108.5 \\
\hline 7 & 164.1 & 163.9 & 164.1 & 164.2 & 164.2 & 164.1 & 162.6 & 163.2 & 162.7 & 163.2 & 161.3 & 160.9 & 161.3 & 161.3 & 161.3 \\
\hline 8 & 98.2 & 98.2 & 93.7 & 93.6 & 93.6 & 93.6 & 104.6 & 93.6 & 104.0 & 93.5 & 103.3 & 103.3 & 103.9 & 105.2 & 104.3 \\
\hline 9 & 156.8 & 160.7 & 156.3 & 157.3 & 156.4 & 156.5 & 156.0 & 156.2 & 156.0 & 156.2 & 155.2 & 155.1 & 155.2 & 155.2 & 154.4 \\
\hline 10 & 104.0 & 103.0 & 104.0 & 104.1 & 104.0 & 104.0 & 104.1 & 103.4 & 104.6 & 103.4 & 102.9 & 102.8 & 102.8 & 103.8 & 103.1 \\
\hline $1^{\prime}$ & 120.9 & 122.0 & 120.9 & 121.1 & 121.2 & 121.6 & 121.6 & 121.1 & 122.0 & 121.4 & 121.5 & 121.9 & 121.6 & 121.6 & 121.5 \\
\hline $2^{\prime}$ & 130.9 & 115.1 & 130.9 & 115.7 & 115.2 & 115.3 & 128.9 & 128.5 & 114.0 & 113.3 & 129.1 & 114.3 & 114.3 & 129.2 & 128.8 \\
\hline $3^{\prime}$ & 115.1 & 145.0 & 115.1 & 145.2 & 144.9 & 144.8 & 115.8 & 116.0 & 145.8 & 145.7 & 115.9 & 145.9 & 145.9 & 115.9 & 115.8 \\
\hline $4^{\prime}$ & 159.9 & 147.7 & 159.9 & 148.4 & 148.5 & 148.4 & 161.1 & 160.6 & 150.0 & 149.7 & 160.9 & 149.8 & 149.9 & 161.1 & 161.3 \\
\hline $5^{\prime}$ & 115.1 & 115.6 & 115.1 & 115.5 & 116.0 & 116.3 & 115.8 & 116.0 & 115.7 & 116.0 & 115.7 & 115.9 & 116.4 & 115.7 & 115.9 \\
\hline $6^{\prime}$ & 130.9 & 120.0 & 130.9 & 121.1 & 122.2 & 121.2 & 128.9 & 128.5 & 119.4 & 119.0 & 128.8 & 119.5 & 119.7 & 129.2 & 128.8 \\
\hline $1^{\prime \prime}$ & 101.4 & & 100.9 & 101.9 & 101.9 & 101.2 & 78.7 & 73.1 & 73.4 & 73.0 & 74.2 & 74.1 & 74.2 & 74.3 & 73.9 \\
\hline $2^{\prime \prime}$ & 74.2 & & 74.2 & 70.4 & 71.3 & 74.1 & 73.4 & 70.6 & 73.4 & 70.6 & 72.1 & 70.8 & 70.1 & 68.4 & 71.3 \\
\hline $3^{\prime \prime}$ & 76.4 & & 77.5 & 70.6 & 73.3 & 76.5 & 70.9 & 78.9 & 78.8 & 78.9 & 78.9 & 78.9 & 73.8 & 73.9 & 78.9 \\
\hline $4^{\prime \prime}$ & 69.9 & & 69.9 & 71.2 & 68.0 & 70.6 & 70.6 & 70.3 & 70.7 & 70.2 & 70.6 & 70.7 & 68.4 & 69.9 & 70.9 \\
\hline $5^{\prime \prime}$ & 75.7 & & 76.4 & 70.1 & 75.9 & 76.0 & 81.8 & 81.5 & 82.0 & 81.5 & 82.0 & 80.9 & 69.5 & 70.6 & 81.3 \\
\hline $6^{\prime \prime}$ & 66.9 & & 60.9 & 17.5 & 60.2 & 67.0 & 61.3 & 61.5 & 61.7 & 61.5 & 61.4 & 61.4 & & & 60.8 \\
\hline $1^{\prime \prime \prime}$ & 100.7 & & & & & 100.8 & & & & & 73.5 & 73.5 & 73.2 & 73.4 & 75.0 \\
\hline $2^{\prime \prime \prime}$ & 70.3 & & & & & 70.1 & & & & & 71.2 & 71.9 & 71.8 & 71.2 & 70.1 \\
\hline $3^{\prime \prime \prime}$ & 70.6 & & & & & 70.4 & & & & & 77.9 & 77.9 & 78.9 & 78.9 & 73.9 \\
\hline $4^{\prime \prime \prime}$ & 71.8 & & & & & 71.9 & & & & & 69.1 & 70.8 & 70.8 & 70.8 & 70.1 \\
\hline $5^{\prime \prime \prime}$ & 68.2 & & & & & 68.3 & & & & & 81.0 & 80.9 & 82.0 & 81.9 & 70.9 \\
\hline $6^{\prime \prime \prime}$ & 17.7 & & & & & 17.7 & & & & & 60.0 & 61.7 & 61.5 & 61.2 & \\
\hline
\end{tabular}

Note: ${ }^{13} \mathrm{C}-\mathrm{NMR}$ data $(\delta)$ were measured in DMSO- $\mathrm{d}_{6}$ at $100 \mathrm{MHz}$.

Rutin (6): ESI-MS m/z: 611[M+H] ${ }^{+} .{ }^{1} \mathrm{H}-\mathrm{NMR}$ (400 MHz, DMSO-d $\left.{ }_{6}\right) \delta: 12.59$ (1H, s, OH-5), 10.85 $(1 \mathrm{H}, \mathrm{s}, \mathrm{OH}-7), 9.69(1 \mathrm{H}, \mathrm{s}, \mathrm{OH}-4$ '), $9.20(1 \mathrm{H}, \mathrm{s}, \mathrm{OH}-$ 3'), $7.54\left(1 \mathrm{H}, \mathrm{d}, J=2.0 \mathrm{~Hz}, \mathrm{H}-2^{\prime}\right), 7.53\left(1 \mathrm{H}, \mathrm{s}, \mathrm{H}-6^{\prime}\right)$, $6.83\left(1 \mathrm{H}, \mathrm{d}, J=8.4 \mathrm{~Hz}, \mathrm{H}-5^{\prime}\right), 6.38(1 \mathrm{H}, \mathrm{d}, J=1.2 \mathrm{~Hz}$, $\mathrm{H}-8), 6.20(1 \mathrm{H}, \mathrm{d}, J=1.2 \mathrm{~Hz}, \mathrm{H}-6), 5.34(1 \mathrm{H}, \mathrm{d}$, $J=6.8 \mathrm{~Hz}, \mathrm{Glu}-\mathrm{H}-1), 4.38(1 \mathrm{H}, \mathrm{s}$, Rha-H-1), 0.99 $(3 \mathrm{H}, \mathrm{d}, J=6.0 \mathrm{~Hz}$, Rha-H- 6$) ;{ }^{13} \mathrm{C}-\mathrm{NMR}(100 \mathrm{MHz}$, DMSO- $\left.\mathrm{d}_{6}, \delta_{c}\right)$ See Table 2 . These data were in consistent with those of rutin (22).

Vitexin (7): ESI-MS m/z: 433[M+H] $]^{+}{ }^{1} \mathrm{H}-\mathrm{NMR}$ (400 MHz, DMSO-d ${ }_{6}$ ) $\delta: 13.17(1 \mathrm{H}, \mathrm{s}, 5-\mathrm{OH}), 10.85$ $(1 \mathrm{H}, \mathrm{s}, 7-\mathrm{OH}), 10.37\left(1 \mathrm{H}, \mathrm{s}, 4^{\prime}-\mathrm{OH}\right), 8.03(2 \mathrm{H}, \mathrm{d}$, $\left.J=8.4 \mathrm{~Hz}, \mathrm{H}-2^{\prime}, 6^{\prime}\right), 6.89\left(2 \mathrm{H}, \mathrm{d}, J=8.4 \mathrm{~Hz}, \mathrm{H}-3^{\prime}\right.$, $\left.5^{\prime}\right), 6.78(1 \mathrm{H}, \mathrm{s}, \mathrm{H}-3), 6.27(1 \mathrm{H}, \mathrm{s}, \mathrm{H}-6), 4.68(1 \mathrm{H}$, d, $J=10.0 \mathrm{~Hz}$, Glu-H-1); ${ }^{13} \mathrm{C}-\mathrm{NMR}(100 \mathrm{MHz}$,
DMSO- $\mathrm{d}_{6}, \delta_{\mathrm{c}}$ ) See Table 2 . These data were in consistent with those of vitexin (23).

Isovitexin (8): $\mathrm{ESI}-\mathrm{MS} \mathrm{m} / \mathrm{z}: 433[\mathrm{M}+\mathrm{H}]^{+}$. ${ }^{1} \mathrm{H}-\mathrm{NMR}(400 \mathrm{MHz}$, DMSO-d $)$ ): $13.55(1 \mathrm{H}, \mathrm{s}$, $5-\mathrm{OH}), 10.38\left(1 \mathrm{H}, \mathrm{s}, 4^{\prime}-\mathrm{OH}\right), 7.93(2 \mathrm{H}, \mathrm{d}, J=8.8 \mathrm{~Hz}$, H-2', 6'), $6.91\left(2 \mathrm{H}, \mathrm{d}, J=8.8 \mathrm{~Hz}, \mathrm{H}-3^{\prime}, 5^{\prime}\right), 6.78(1 \mathrm{H}$, s, H-3), $6.52(1 \mathrm{H}, \mathrm{s}, \mathrm{H}-8), 4.60(1 \mathrm{H}, \mathrm{d}, J=9.6 \mathrm{~Hz}$, Glu-H-1); ${ }^{13} \mathrm{C}-\mathrm{NMR}\left(100 \mathrm{MHz}\right.$, DMSO-d ${ }_{6}, \delta_{\mathrm{c}}$ ) See Table 2. These data were in consistent with those of isovitexin (24).

Orientin (9): ESI-MS m/z: $449[\mathrm{M}+\mathrm{H}]^{+}$. ${ }^{1} \mathrm{H}-\mathrm{NMR}\left(400 \mathrm{MHz}\right.$, DMSO-d $\left.{ }_{6}\right) \delta: 13.17(1 \mathrm{H}, \mathrm{s}, 5-\mathrm{OH})$, $10.56(1 \mathrm{H}, \mathrm{s}, 7-\mathrm{OH}), 10.04\left(1 \mathrm{H}, \mathrm{s}, 3^{\prime}-\mathrm{OH}\right), 9.22(1 \mathrm{H}, \mathrm{s}$, 4'- $\mathrm{OH}$ ), 7.53 (1H, d, J = 8.4 Hz, H-6'), 7.48 (H, brs, H-2'), 6.86 (1H, d, J = $\left.8.4 \mathrm{~Hz}, \mathrm{H}-5^{\prime}\right), 6.64$ (1H, s, H-3), $6.26(1 \mathrm{H}, \mathrm{s}, \mathrm{H}-6), 4.68(1 \mathrm{H}, \mathrm{d}, \mathrm{J}=10.0 \mathrm{~Hz}$, Glu-H-1); 
${ }^{13} \mathrm{C}-\mathrm{NMR}\left(100 \mathrm{MHz}, \mathrm{DMSO}-\mathrm{d}_{6}, \delta_{\mathrm{c}}\right.$ ) See Table 2. These data were in consistent with those of orientin (25).

Isoorientin (10): ESI-MS m/z: $449[\mathrm{M}+\mathrm{H}]^{+}$. ${ }^{1} \mathrm{H}-\mathrm{NMR}\left(400 \mathrm{MHz}, \mathrm{DMSO}-\mathrm{d}_{6}\right) \delta: 13.56(1 \mathrm{H}, \mathrm{s}$, 5-OH), $10.53(1 \mathrm{H}, \mathrm{s}, 7-\mathrm{OH}), 9.65(1 \mathrm{H}, \mathrm{s}, 3$ '-OH), 9.47(1H, s, 4'-OH), 7.42 (1H, d, J = 8.4 Hz, H-6'), $7.40\left(1 \mathrm{H}\right.$, brs, H-2'), $6.89\left(1 \mathrm{H}, \mathrm{d}, \mathrm{J}=8.0 \mathrm{~Hz}, \mathrm{H}-5^{\prime}\right)$, $6.68(1 \mathrm{H}, \mathrm{s}, \mathrm{H}-3), 6.48(1 \mathrm{H}, \mathrm{s}, \mathrm{H}-8), 4.58(1 \mathrm{H}, \mathrm{d}$, $\mathrm{J}=10.0 \mathrm{~Hz}$, Glu-H-1); ${ }^{13} \mathrm{C}-\mathrm{NMR}(100 \mathrm{MHz}$, DMSO- $\left.d_{6}, \delta_{c}\right)$ See Table 2 . These data were in consistent with those of isoorientin (26).

Vicenin-2 (11): ESI-MS m/z 596[M+H $]^{+}$. ${ }^{1} \mathrm{HNMR}\left(400 \mathrm{MHz}, \mathrm{DMSO}-\mathrm{d}_{6}\right) \delta: 13.71(1 \mathrm{H}, \mathrm{s}, \mathrm{OH}-$ 5), 10.39 (1H, s, OH-7), 9.33 (1H, s, OH-4'), 8.05 $\left(2 \mathrm{H}, \mathrm{d}, J=8.8 \mathrm{~Hz}, 2 \mathrm{H}, \mathrm{H}-2^{\prime}, 6^{\prime}\right), 6.98(\mathrm{~d}, J=8.8 \mathrm{~Hz}$, $\left.2 \mathrm{H}, \mathrm{H}-3^{\prime}, 5^{\prime}\right), 6.85$ (1H, s, H-3); ${ }^{13} \mathrm{C}-\mathrm{NMR}(100 \mathrm{MHz}$, DMSO- $\left.\mathrm{d}_{6}, \delta_{c}\right)$ See Table 2 . These data were in consistent with those of vicenin-2 (27).

Lucenin-2 (12): ESI-MS m/z: $611[\mathrm{M}+\mathrm{H}]^{+}$. ${ }^{1} \mathrm{H}-\mathrm{NMR}(400 \mathrm{MHz}, \mathrm{DMSO}-\mathrm{d} 6) \delta: 13.71(1 \mathrm{H}, \mathrm{s}$, OH-5), 9.29 (2H, s, 3', 4'-OH), 7.54 (1H, d, $J=8.4 \mathrm{~Hz}$, H-6'), 7.48 (1H, s, H-2'), 6.88 (1H, d, $J=8.0 \mathrm{~Hz}$, H-5'), 6.69 (1H, s, H-3), $4.81(1 \mathrm{H}, \mathrm{d}, J=9.6 \mathrm{~Hz}$, 6-Glu-H-1), 4.76 (1H, d, $J=9.6 \mathrm{~Hz}, 8-\mathrm{Glu}-\mathrm{H}-1)$; ${ }^{13} \mathrm{C}-\mathrm{NMR}\left(100 \mathrm{MHz}, \mathrm{DMSO}-\mathrm{d}_{6}, \delta_{\mathrm{c}}\right.$ ) See Table 2. These data were in consistent with those of lucenin-2 (28).

Lucenin-1 (13): ESI-MS m/z: $581[\mathrm{M}+\mathrm{H}]^{+}$. ${ }^{1} \mathrm{H}-\mathrm{NMR}(400 \mathrm{MHz}, \mathrm{DMSO}-\mathrm{d} 6) \delta: 13.71(1 \mathrm{H}, \mathrm{s}$, OH-5), 9.29 (2H, s, 3', 4'-OH), $7.52(1 \mathrm{H}, \mathrm{d}, J=8.0 \mathrm{~Hz}$, H-6'), 7.47 (1H, d, $J=2.0 \mathrm{~Hz}, \mathrm{H}-2$ '), $6.87(1 \mathrm{H}, \mathrm{d}$, $J=8.0 \mathrm{~Hz}, \mathrm{H}-5$ '), 6.66 (1H, s, H-3), 4.75 (1H, d,
$J=10.0 \mathrm{~Hz}$, Glu-H-1), $4.69(1 \mathrm{H}, \mathrm{d}, J=9.6 \mathrm{~Hz}$, Xyl-H-1); ${ }^{13} \mathrm{C}-\mathrm{NMR}\left(100 \mathrm{MHz}, \mathrm{DMSO}-\mathrm{d}_{6}, \delta_{\mathrm{c}}\right.$ ) See Table 2. These data were in consistent with those of lucenin-1 (29-30).

Vicenin-1 (14): ESI-MS m/z: $565[\mathrm{M}+\mathrm{H}]^{+}$. ${ }^{1} \mathrm{H}-\mathrm{NMR}(400 \mathrm{MHz}, \mathrm{DMSO}-\mathrm{d} 6) \delta: 13.66(1 \mathrm{H}, \mathrm{s}$, OH-5), 8.02 (2H, d, $J=8.4$ Hz, H-2',6'), 6.89 (2H, $\mathrm{d}, J=8.6 \mathrm{~Hz}, \mathrm{H}-3$ ', 5'), $6.81(1 \mathrm{H}, \mathrm{s}, \mathrm{H}-3), 4.81(1 \mathrm{H}$, $\mathrm{d}, J=10.0 \mathrm{~Hz}$, Glu-H-1), $4.73(1 \mathrm{H}, \mathrm{d}, J=10.0 \mathrm{~Hz}$, Xyl-H-1); ${ }^{13} \mathrm{C}-\mathrm{NMR}$ (100 MHz, DMSO-d,$\delta_{c}$ ) See Table 2. These data were in consistent with those of vicenin-1 (31).

Vicenin-3 (15): ESI-MS m/z: $565[\mathrm{M}+\mathrm{H}]^{+}$. ${ }^{1} \mathrm{H}-\mathrm{NMR}\left(400 \mathrm{MHz}, \mathrm{DMSO}-\mathrm{d}_{6}\right) \delta: 13.66(1 \mathrm{H}, \mathrm{s}$, OH-5), 8.01 (2H, d, $J=9.6 \mathrm{~Hz}, \mathrm{H}-2$ ', 6') $6.91(2 \mathrm{H}$, $\mathrm{d}, J=8.8 \mathrm{~Hz}, \mathrm{H}-3$ ', 5'), $6.81(1 \mathrm{H}, \mathrm{s}, \mathrm{H}-3), 4.82(1 \mathrm{H}$, $\mathrm{d}, J=9.6 \mathrm{~Hz}$, Glu-H-1), $4.71(1 \mathrm{H}, \mathrm{d}, J=10.0 \mathrm{~Hz}$, Xyl-H-1); ${ }^{13} \mathrm{C}-\mathrm{NMR}\left(100 \mathrm{MHz}, \mathrm{DMSO}-\mathrm{d}_{6}, \delta_{c}\right)$ See Table 2. These data were in consistent with those of vicenin-3 (32).

\section{Determination of antioxidant activity in vitro}

Flavonoids showed various biological functions with low toxicity. The diverse biological functions were considered to be related with their strong antioxidant activities (33-35). Therefore, the antioxidant activities of the flavone compounds 1-15 were measured by DPPH, ABTS and FRAP assays, respectively, while ascorbic acid was used as the positive control. Most compounds exhibited strong antioxidant activities, the $\mathrm{IC}_{50}$ and FRAP values were given in Table 3. The results showed that compounds 2 ,

Table 3. Antioxidant activities of compound 1-15.

\begin{tabular}{|c|c|c|c|}
\hline Compound & $\left.\mathrm{DPPH} \mathrm{IC}_{50} / \mu \mathrm{g} / \mathrm{L}\right)$ & $\mathrm{ABTS}^{\cdot+} \mathrm{IC}_{50} /(\mu \mathrm{g} / \mathrm{L})$ & 1.02 \\
\hline 1 & $>100$ & $>100$ & 6.84 \\
\hline 2 & 6.96 & 5.42 & 0.70 \\
\hline 3 & $>100$ & $>100$ & 6.99 \\
\hline 4 & 11.64 & 8.01 & 5.57 \\
\hline 5 & 11.67 & 7.23 & 8.31 \\
\hline 6 & 13.79 & 9.65 & 0.77 \\
\hline 7 & $>100$ & $>100$ & 0.91 \\
\hline 8 & $>100$ & $>100$ & 9.80 \\
\hline 9 & 19.85 & 4.22 & 4.25 \\
\hline 10 & 10.27 & 9.63 & 3.89 \\
\hline 11 & 87.91 & 29.44 & 4.69 \\
\hline 12 & 25.08 & 12.68 & 5.32 \\
\hline 13 & 25.43 & 10.43 & 2.46 \\
\hline 14 & 98.83 & 89.43 & 1.84 \\
\hline 15 & $>100$ & 98.17 & 3.21 \\
\hline Ascorbic acid & 12.43 & 5.89 & \\
\hline
\end{tabular}


Table 4. The condition of MS optimization for MRM mode.

\begin{tabular}{|c|c|c|c|c|c|}
\hline Compound & $\begin{array}{c}\text { Retention time } \\
(\mathrm{min})\end{array}$ & $\begin{array}{c}{[\mathrm{M}-\mathrm{H}]^{-}} \\
(\mathrm{m} / \mathrm{z})\end{array}$ & $\begin{array}{c}\text { Quantitative ions } \\
(\mathrm{m} / \mathrm{z})\end{array}$ & $\begin{array}{c}\text { Declustering potential } \\
\mathrm{eV}\end{array}$ & $\begin{array}{c}\text { Collision energy } \\
\mathrm{eV}\end{array}$ \\
\hline 2 & 6.54 & 300.9 & 150.9 & -140 & -30 \\
\hline 4 & 5.63 & 447.0 & 299.5 & -120 & -36 \\
\hline 5 & 5.33 & 463.0 & 299.8 & -130 & -37 \\
\hline 6 & 5.06 & 609.0 & 299.8 & -100 & -50 \\
\hline 9 & 4.61 & 447.0 & 326.5 & -200 & -33 \\
\hline 10 & 3.47 & 447.1 & 356.8 & -60 & -35 \\
\hline 11 & 3.06 & 592.9 & 352.8 & -180 & -50 \\
\hline 12 & 2.74 & 609.0 & 368.7 & -160 & -50 \\
\hline 13 & 3.13 & 579.1 & 368.8 & -195 & -50 \\
\hline 14 & 3.64 & 562.9 & 382.9 & -180 & -48 \\
\hline
\end{tabular}

4-6, 9-14 had more significant antioxidative effects compared with the others. The successive order of $\mathrm{IC}_{50}$ values of DPPH radical scavenging activity was as follows: $2>10>4>5>6>9>12>13>11>$ 14 ; The successive order of $\mathrm{IC}_{50}$ values of ABTS radical scavenging activity was as follows: $9>2>5>4$ $>10>6>13>12>11>14$; The successive order of FRAP values of total antioxidant capacity was as follows: $9>6>4>2>5>13>12>10>11>14$.

The presence of 3', 4'-dihydroxy group (catechol) in the $\mathrm{B}$ ring and/or the presence of the $3-\mathrm{OH}$ group in the $\mathrm{C}$ ring is often deemed to be essential for radical scavenging activity of flavonoids. In addition, the 5-OH group in combination with 1, 4-pyrone moiety, and $\mathrm{C} 2=\mathrm{C} 3$ double bond may increase the radical scavenging activity (36-38). The fifteen compounds were divided into four categories (Figure 1) by the chemical structure: quercetin derivatives (compound $2,4,5,6$ ), apigenin derivatives (compound 7, 8, 11, 14, 15), kaempferol derivatives (compound 13 ) and

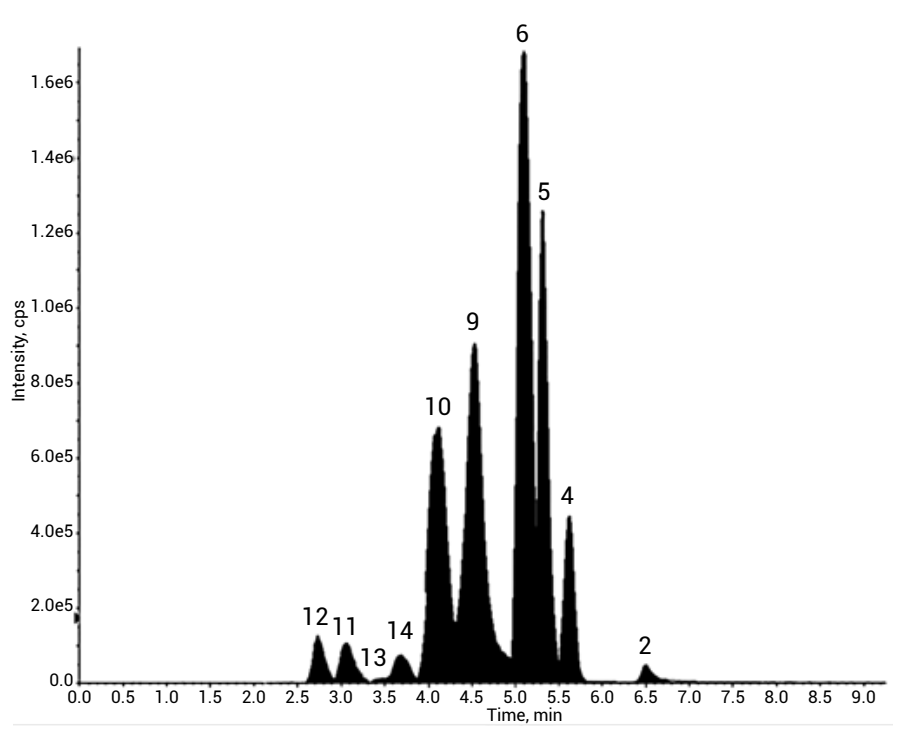

luteolin derivatives (compound 9, 10, 12, 13). The luteolin and quercetin derivatives had significantly higher oxidation resistances than the kaempferol and apigenin derivatives due to the ortho-diphenol structure at 3', 4' position in the B ring. Quercetin derivatives had higher antioxidant activities than luteolin derivatives on the whole, possibly due to the presence of a 3-OH group in the $\mathrm{C}$ ring. Compared with the quercetin and luteolin derivatives, apigenin and kaempferol derivatives showed weaker antioxidant activities. So, compounds 2, 4-6, and 9-14 were selected as the antioxidant substance in THL, and an analytical method to determine these antioxidants simultaneously by using ultra-performance liquid chromatography coupled with triple-quadrupole tandem mass spectrometry was then explored.

\section{Analytical method optimization}

The Mass spectra in positive and negative ion modes were compared. It was found that the flavonoids had higher sensitivity in the negative ion mode, which made it easier to confirm molecular ions or protonated molecule ions in the identification of each peak. Therefore, the mass spectrometer was set to collect data in negative ion mode. All MS optimization experiments were carried out in MS scan mode and in product scan mode. Quantitative analysis was carried out in MRM mode. The tune page parameters and conditions for each of the MRM transitions were optimized by infusing the neat standard solution into the mass spectrometer. Each compound was

Figure 2. Total ion chromatograms for standards mixture of THL. 
Table 5. Calibration curves, linear ranges, correlation coefficient, LOQ, and LOD of ten compounds.

\begin{tabular}{|c|c|c|c|c|c|}
\hline Compound & $\begin{array}{c}\text { Regression equation } \\
(\mathrm{n}=6)\end{array}$ & $\begin{array}{c}\text { Linear range } \\
\mathrm{ng} / \mathrm{mL}\end{array}$ & $R^{2}$ & $\begin{array}{c}\text { LOQ } \\
\mathrm{ng} / \mathrm{mL}\end{array}$ & $\begin{array}{c}\text { LOD } \\
\mathrm{ng} / \mathrm{mL}\end{array}$ \\
\hline 2 & $\mathrm{Y}=679.15 \mathrm{X}-4.99 \times 10^{4}$ & $5.0-500.0$ & 0.9986 & 3.50 & 1.05 \\
\hline 4 & $\mathrm{Y}=4454.90 \mathrm{X}-1.91 \times 10^{5}$ & $5.0-500.0$ & 0.9980 & 2.35 & 0.71 \\
\hline 5 & $\mathrm{Y}=10979.57 \mathrm{X}+2.18 \times 10^{4}$ & $5.0-500.0$ & 0.9996 & 1.12 & 0.34 \\
\hline 6 & $\mathrm{Y}=17346.63 \mathrm{X}-3.08 \times 10^{4}$ & $5.0-1000.0$ & 0.9995 & 1.02 & 0.31 \\
\hline 9 & $\mathrm{Y}=14472.60 \mathrm{X}-3.64 \times 10^{5}$ & $5.0-1000.0$ & 0.9998 & 1.87 & 0.56 \\
\hline 10 & $\mathrm{Y}=7102.75 \mathrm{X}-1.20 \times 10^{5}$ & $5.0-2000.0$ & 0.9999 & 2.15 & 0.65 \\
\hline 11 & $\mathrm{Y}=1192.95 \mathrm{X}-1.29 \times 10^{4}$ & $5.0-2000.0$ & 0.9999 & 2.65 & 0.80 \\
\hline 12 & $\mathrm{Y}=1318.13 \mathrm{X}-2.46 \times 10^{4}$ & $5.0-2000.0$ & 0.9998 & 2.76 & 0.83 \\
\hline 13 & $\mathrm{Y}=811.16 \mathrm{X}-7.01 \times 10^{3}$ & $5.0-1000.0$ & 0.9999 & 3.06 & 0.92 \\
\hline 14 & $\mathrm{Y}=1212.76 \mathrm{X}-2.86 \times 10^{4}$ & $5.0-2000.0$ & 0.9998 & 2.87 & 0.86 \\
\hline
\end{tabular}

Table 6. The precision, repeatability, stability, and recovery in UPLC-MS/MS analysis for the ten compounds in samples.

\begin{tabular}{|c|c|c|c|c|c|c|}
\hline \multirow{2}{*}{ Compound } & \multicolumn{2}{|c|}{ Precision RSD (\%) } & \multirow{2}{*}{$\begin{array}{c}\text { Repeatability } \\
(\mathrm{RSD}, \%, \mathrm{n}=6)\end{array}$} & \multirow{2}{*}{$\begin{array}{c}\text { Stability } \\
(\mathrm{RSD}, \%, \mathrm{n}=6)\end{array}$} & \multicolumn{2}{|c|}{ Recovery $(\%, \mathrm{n}=3)$} \\
\hline & Intraday $(n=6)$ & Interday $(\mathrm{n}=6)$ & & & Mean & RSD \\
\hline 2 & 1.66 & 3.47 & 3.51 & 2.12 & 96.39 & 3.44 \\
\hline 4 & 1.89 & 2.53 & 3.47 & 3.44 & 99.27 & 2.97 \\
\hline 5 & 2.07 & 2.53 & 2.68 & 1.73 & 95.77 & 4.26 \\
\hline 6 & 1.53 & 1.77 & 3.55 & 2.76 & 104.84 & 3.67 \\
\hline 9 & 2.34 & 2.49 & 2.94 & 3.58 & 102.73 & 2.54 \\
\hline 10 & 1.55 & 2.05 & 1.97 & 2.91 & 101.89 & 4.01 \\
\hline 11 & 1.93 & 2.36 & 2.86 & 3.55 & 98.45 & 4.37 \\
\hline 12 & 2.84 & 3.09 & 3.84 & 3.86 & 103.26 & 2.88 \\
\hline 13 & 2.45 & 2.56 & 2.65 & 1.94 & 100.78 & 3.89 \\
\hline 14 & 2.64 & 2.48 & 3.83 & 2.68 & 97.26 & 2.47 \\
\hline
\end{tabular}

studied individually to achieve the best scan time, Declustering Potential (DP), and Collision Energy (CE). The MRM transitions for the tested compounds were shown in Table 4, and the total ion chromatogram of ten markers was shown in Figure 2.

\section{Analytical method validation}

A fast and sensitive UPLC-MS/MS method was developed for the simultaneous quantification of ten compounds in THL. The method for quantitative analysis was validated by determining the linearity, intra-day and inter-day precisions, LOD and LOQ, stability, and accuracy. The calibration curves were prepared by injecting standard solutions at seven concentration levels and showed good linearity in the test ranges, ranging from 0.9980 to 0.9999 (Table 5). The overall LODs and LOQs were in the range of $0.31-1.05 \mathrm{ng} / \mathrm{mL}$ and $1.02-3.50 \mathrm{ng} / \mathrm{mL}$, respectively. The overall recoveries ranged from $95.77 \%$ to $104.84 \%$ with RSD less than 5\%. The RSD values of stability and repeatability, intra-day and inter-day variations of the ten analytes were all less than $4 \%$ (Table 6 ). The overall validation results showed that the UPLC-MS/MS method has good linearity, accuracy, precision, and sensitivity, and could be used for the quantification analysis of antioxidants in THL.

\section{Quantitative analysis of a sample}

The proposed UPLC-MS/MS method was subsequently applied to determine the ten compounds' content in THL from the different habitats. The results were shown in Table 7. It can be seen from Table 7 that the contents of the ten major antioxidant components in THL from different habitats were very different. Besides, some compounds were undetectable in some batches due to the content below the LOD, which might be related to the different germplasm resources of $T$. hemsleyanum and its environmental diversity of different planting bases. The contents of quercetin, quercitrin, and isoquercitrin in samples from different habitats were all lower than $100 \mu \mathrm{g} / \mathrm{g}$. The contents of rutin in THL from 


\begin{tabular}{|c|c|c|c|c|c|c|c|c|c|c|c|c|c|c|c|c|c|}
\hline$\Xi$ & 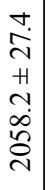 & 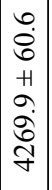 & $\mid \begin{array}{l}n \\
2 \\
+1 \\
0 \\
\dot{a} \\
\dot{8} \\
n\end{array}$ & 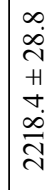 & 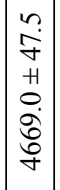 & 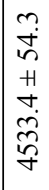 & 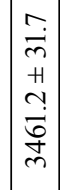 & $\mid \begin{array}{c}n \\
0 \\
+ \\
n \\
\tilde{\dot{d}} \\
\end{array}$ & $\begin{array}{l}\tilde{y} \\
0 \\
+1 \\
\text { o. }\end{array}$ & $\mid \begin{array}{c}0 \\
0 \\
+1 \\
\dot{\omega}\end{array}$ & $\left|\begin{array}{l}0 \\
\tilde{D} \\
+1 \\
\tilde{i} \\
\end{array}\right|$ & - & $\left|\begin{array}{c}\tilde{c} \\
\tilde{n} \\
\infty \\
\infty \\
\alpha \\
\alpha\end{array}\right|$ & $\left|\begin{array}{c}\infty \\
\sigma \\
+ \\
\dot{+} \\
\dot{\sigma}\end{array}\right|$ & $\left|\begin{array}{l}0 \\
0 \\
H \\
\tilde{\sigma} \\
\sigma\end{array}\right|$ & - & 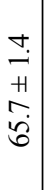 \\
\hline 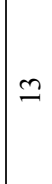 & $\begin{array}{l}+ \\
\infty \\
+ \\
+1 \\
\sim \\
\qquad \begin{array}{l} \\
+\end{array}\end{array}$ & 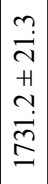 & 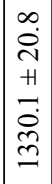 & $\begin{array}{l}0 \\
\tilde{+} \\
+1 \\
\alpha \\
\tilde{\sigma} \\
\tilde{\sigma}\end{array}$ & 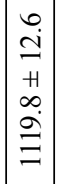 & 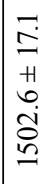 & $\mid \begin{array}{c}\tilde{\sigma} \\
+ \\
\dot{\theta} \\
\dot{\delta} \\
\varrho\end{array}$ & 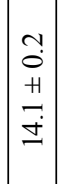 & - & \begin{tabular}{|l}
- \\
0 \\
+1 \\
0 \\
$i$ \\
$i$
\end{tabular} & $\mid \begin{array}{l}\infty \\
\dot{i} \\
\dot{H} \\
\hat{j} \\
\dot{\gamma}\end{array}$ & - & $\left|\begin{array}{l}\tilde{y} \\
0 \\
+1 \\
\tilde{z} \\
\dot{I}\end{array}\right|$ & $\mid \begin{array}{c}n \\
i \\
+1 \\
i \\
\tilde{g} \\
0\end{array}$ & & & 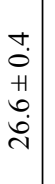 \\
\hline$\cong$ & 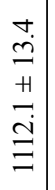 & 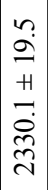 & 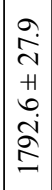 & 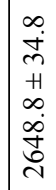 & 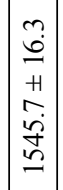 & 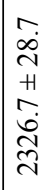 & 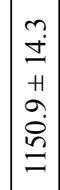 & $\begin{array}{l}r \\
\hat{0} \\
+ \\
\hat{i} \\
\hat{i}\end{array}$ & $\begin{array}{l}- \\
0 \\
+1 \\
\sim \\
\sigma\end{array}$ & $\begin{array}{l}0 \\
0 \\
+1 \\
n \\
\sigma \\
\sigma\end{array}$ & 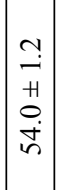 & - & $\mid \begin{array}{l}-1 \\
\dot{H} \\
+ \\
0 \\
0 \\
\dot{m}\end{array}$ & $\left|\begin{array}{l}\infty \\
\dot{D} \\
+ \\
i \\
\dot{0} \\
\infty\end{array}\right|$ & $\left|\begin{array}{l}0 \\
0 \\
0 \\
0 \\
\infty \\
\infty \\
\infty \\
n\end{array}\right|$ & $\begin{array}{l}\infty \\
\dot{0} \\
+ \\
\stackrel{1}{I} \\
\dot{I}\end{array}$ & $\begin{array}{l}\dot{0} \\
\dot{+} \\
+ \\
\hat{o} \\
\dot{\sigma}\end{array}$ \\
\hline$=$ & $\begin{array}{l}\tilde{y} \\
\vec{m} \\
+1 \\
\infty \\
\dot{m} \\
\vec{v}\end{array}$ & $\mid \begin{array}{l}m \\
\infty \\
0 \\
+ \\
+ \\
+ \\
\dot{0} \\
\dot{N}\end{array}$ & 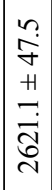 & $\begin{array}{l}m \\
\tilde{\delta} \\
+1 \\
0 \\
0 \\
\infty \\
\tilde{y} \\
\hat{\gamma}\end{array}$ & 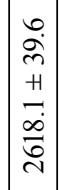 & 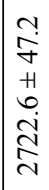 & 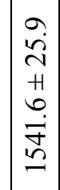 & $\begin{array}{l}0 \\
0 \\
0 \\
+1 \\
0 \\
0 \\
0\end{array}$ & $\begin{array}{l}3 \\
0 \\
+1 \\
0 \\
0 \\
0\end{array}$ & $\begin{array}{l}\tilde{1} \\
\stackrel{1}{0} \\
\ddot{0} \\
\stackrel{0}{0}\end{array}$ & 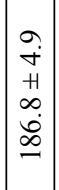 & - & 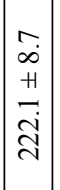 & 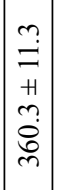 & $\begin{array}{l}\tilde{n} \\
\dot{b} \\
+1 \\
\tilde{\mathscr{b}} \\
\infty \\
-\end{array}$ & 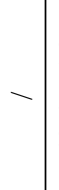 & $\begin{array}{l}3 \\
\vec{i} \\
+ \\
\dot{y} \\
\dot{y}\end{array}$ \\
\hline ㅇ & $\begin{array}{l}a \\
i \\
+1 \\
+ \\
\dot{0} \\
\dot{0} \\
\dot{n}\end{array}$ & 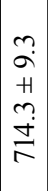 & $\begin{array}{l}\infty \\
\dot{+} \\
+ \\
\dot{0} \\
\dot{+} \\
\dot{+}\end{array}$ & 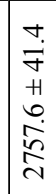 & 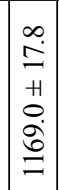 & 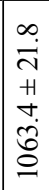 & 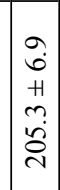 & $\mid \begin{array}{l}n \\
n \\
n \\
0 \\
0 \\
\overrightarrow{0} \\
i\end{array}$ & $\begin{array}{l}-\overrightarrow{0} \\
+ \\
m \\
+\end{array}$ & 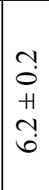 & 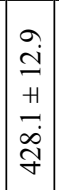 & - & 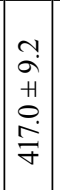 & 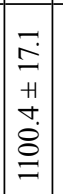 & \begin{tabular}{|c|}
2 \\
$\infty$ \\
+1 \\
$\infty$ \\
$\infty$ \\
$\infty$ \\
$\gamma$ \\
\end{tabular} & $\begin{array}{l}\stackrel{g}{\vec{m}} \\
+ \\
+ \\
+ \\
\dot{f} \\
\stackrel{\sim}{N}\end{array}$ & 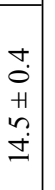 \\
\hline$\sigma$ & 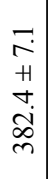 & $\mid \begin{array}{c}0 \\
\infty \\
\infty \\
+1 \\
\sim \\
\infty \\
\infty \\
\sigma \\
\end{array}$ & 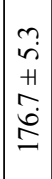 & 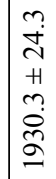 & 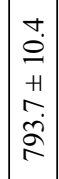 & $\mid \begin{array}{c}2 \\
\stackrel{2}{2} \\
+1 \\
0 \\
\dot{0} \\
\infty\end{array}$ & $\begin{array}{l}0 \\
\dot{n} \\
+ \\
\tilde{b} \\
\dot{\delta} \\
\end{array}$ & 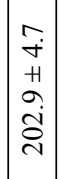 & $\mid \begin{array}{l}-\overrightarrow{0} \\
+ \\
+ \\
\dot{n} \\
\dot{n}\end{array}$ & $\mid \begin{array}{l}\tilde{o} \\
0 \\
H \\
\tilde{\sigma} \\
0\end{array}$ & 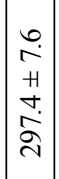 & $\begin{array}{l} \\
\overrightarrow{0} \\
+ \\
\sim \\
\sim \\
+\end{array}$ & 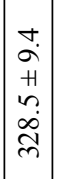 & 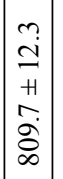 & 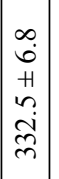 & 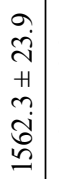 & $\begin{array}{l}m \\
\dot{0} \\
+ \\
\dot{I} \\
\dot{I}\end{array}$ \\
\hline 6 & $\begin{array}{c}\overrightarrow{0} \\
+ \\
\dot{r} \\
\dot{m}\end{array}$ & 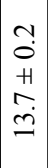 & $\mid \begin{array}{l}\tilde{O} \\
\dot{0} \\
+ \\
\dot{\gamma} \\
\dot{\gamma}\end{array}$ & $\begin{array}{l}7 \\
0 \\
+1 \\
\infty \\
\text { in }\end{array}$ & $\mid \begin{array}{l}\tilde{O} \\
0 \\
H \\
0 \\
\dot{+}\end{array}$ & $\begin{array}{c}\tilde{O} \\
+ \\
+1 \\
\vec{\lambda}\end{array}$ & $\mid \begin{array}{l}\tilde{O} \\
\tilde{H} \\
\tilde{n} \\
\tilde{0} \\
\tilde{0}\end{array}$ & $\begin{array}{l}\hat{0} \\
+ \\
\dot{0} \\
\dot{0}\end{array}$ & 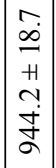 & 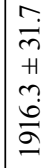 & $\left|\begin{array}{l}\infty \\
0 \\
0 \\
+1 \\
n \\
i \\
i\end{array}\right|$ & 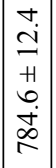 & 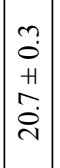 & $\mid \begin{array}{c}0 \\
\dot{0} \\
+1 \\
m \\
\tilde{m} \\
\end{array}$ & $\mid \begin{array}{c}\vec{m} \\
+ \\
+ \\
\vec{b} \\
0\end{array}$ & $\begin{array}{l}n \\
0 \\
+1 \\
0 \\
\dot{0}\end{array}$ & $\begin{array}{l}0 \\
0 \\
0 \\
+1 \\
0 \\
\dot{0} \\
\dot{0} \\
0\end{array}$ \\
\hline in & 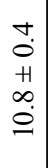 & $\begin{array}{l}-\overrightarrow{0} \\
+1 \\
\stackrel{\theta}{ت}\end{array}$ & 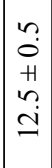 & 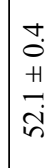 & $\mid \begin{array}{l}\infty \\
0 \\
+ \\
+ \\
+ \\
\dot{\lambda} \\
\mid\end{array}$ & $\begin{array}{l}\vec{j} \\
+ \\
n \\
\text { Cे }\end{array}$ & $\mid \begin{array}{l}0 \\
0 \\
+ \\
\alpha \\
\dot{\infty} \\
\dot{\theta}\end{array}$ & $\mid \begin{array}{c}\infty \\
\dot{\vec{H}} \\
\vec{H} \\
\vec{g}\end{array}$ & $\left|\begin{array}{c}n \\
0 \\
+ \\
n \\
n \\
m\end{array}\right|$ & 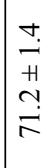 & $\mid \begin{array}{l}0 \\
\dot{0} \\
1 \\
0 \\
\dot{0} \\
\dot{0}\end{array}$ & $\mid \begin{array}{c}0 \\
\dot{+} \\
\dot{H} \\
\stackrel{\sim}{\dot{\gamma}} \\
\mid\end{array}$ & $\mid \begin{array}{l}\infty \\
0 \\
\dot{0} \\
\dot{m} \\
\tilde{m}\end{array}$ & $\left|\begin{array}{l}0 \\
0 \\
+ \\
\dot{m} \\
\tilde{m}\end{array}\right|$ & $\left|\begin{array}{c}\vec{i} \\
+ \\
\vec{j} \\
\vec{n}\end{array}\right|$ & $\begin{array}{l}\infty \\
i \\
+ \\
b \\
\stackrel{i}{i}\end{array}$ & $\begin{array}{l}\text { Fे } \\
\text { m } \\
\approx \\
\text { in }\end{array}$ \\
\hline$\nabla$ & $\mid \begin{array}{l}0 \\
0 \\
+ \\
+ \\
\dot{0} \\
0\end{array}$ & 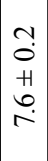 & $\mid \begin{array}{l} \pm \\
0 \\
0 \\
\\
\end{array}$ & $\begin{array}{c}\tilde{O} \\
\tilde{O} \\
+1 \\
\text { J. }\end{array}$ & $\mid \begin{array}{c}c \\
0 \\
+ \\
\infty \\
\infty \\
-\infty\end{array}$ & $\begin{array}{l}3 \\
0 \\
+1 \\
n \\
n\end{array}$ & $\mid \begin{array}{l}\tilde{o} \\
\hat{H} \\
+ \\
\tilde{I} \\
\underline{I}\end{array}$ & $\mid \begin{array}{c}\hat{0} \\
+ \\
\dot{H} \\
\dot{q}\end{array}$ & 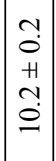 & $\mid \begin{array}{l}n \\
0 \\
H \\
\tilde{O} \\
\tilde{O}\end{array}$ & $\left|\begin{array}{l}\hat{0} \\
+1 \\
\hat{0} \\
\dot{d}\end{array}\right|$ & $\mid \begin{array}{l}-1 \\
0 \\
+1 \\
0 \\
0 \\
0\end{array}$ & 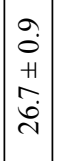 & $\mid \begin{array}{l}\tilde{O} \\
+ \\
+ \\
\dot{\Xi} \\
\Xi\end{array}$ & $\left|\begin{array}{c}\vec{j} \\
\dot{H} \\
i \\
\tilde{b}\end{array}\right|$ & $\begin{array}{l}+ \\
0 \\
+ \\
ث \\
0\end{array}$ & 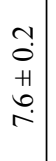 \\
\hline$N$ & $\mid \begin{array}{l}n \\
0 \\
+ \\
n \\
i \\
0\end{array}$ & $\mid \begin{array}{l}2 \\
0 \\
+1 \\
\\
\underline{a}\end{array}$ & $\mid \begin{array}{l}\tilde{u} \\
\hat{H} \\
\tilde{u} \\
\hat{i}\end{array}$ & $\mid \begin{array}{l}\hat{0} \\
+1 \\
\dot{\theta} \\
\dot{\vec{v}}\end{array}$ & 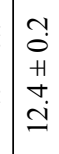 & 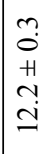 & $\mid \begin{array}{l}t \\
\dot{0} \\
+ \\
\vec{i} \\
\dot{I}\end{array}$ & $\mid \begin{array}{l}\overrightarrow{0} \\
+ \\
\hat{i} \\
\dot{I}\end{array}$ & 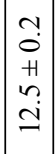 & $\mid \begin{array}{l}\infty \\
0 \\
\dot{+} \\
\dot{I} \\
\dot{I}\end{array}$ & $\mid \begin{array}{l}\tilde{I} \\
\dot{H} \\
\stackrel{+}{i} \\
\underline{I}\end{array}$ & $\mid \begin{array}{l}n \\
0 \\
+ \\
\hat{i} \\
\hat{j}\end{array}$ & 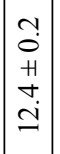 & $\left|\begin{array}{l}n \\
0 \\
+ \\
+ \\
د \\
I\end{array}\right|$ & 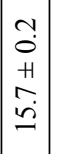 & $\begin{array}{l}0 \\
\dot{0} \\
+ \\
0 \\
\dot{g}\end{array}$ & 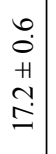 \\
\hline & $\overrightarrow{\dot{n}}$ & $\tilde{r}$ & $\begin{array}{l}\hat{y} \\
\tilde{n}\end{array}$ & 品 & $\mid \begin{array}{l}n \\
b\end{array}$ & in & $\hat{n}$ & $\mid \begin{array}{l}\infty \\
\dot{1} \\
n\end{array}$ & $\mid \begin{array}{l}\hat{\dot{s}} \\
\mid\end{array}$ & $\begin{array}{l}0 \\
\dot{1} \\
\dot{n}\end{array}$ & 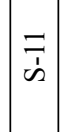 & $\frac{\vec{y}}{\dot{n}}$ & $\left|\begin{array}{l}m \\
\dot{n}\end{array}\right|$ & $\mid \begin{array}{l}\vec{n} \\
\dot{n}\end{array}$ & $\left|\begin{array}{l}n \\
\dot{n}\end{array}\right|$ & $\begin{array}{l}\vec{b} \\
\dot{n}\end{array}$ & $\vec{\prime}$ \\
\hline
\end{tabular}

Yunnan, Guangxi, Guangdong, and Guizhou were greater than other habitats. The contents of vicenin-2, lucenin-2, lucenin-1, and vicenin-1 in THL from Zhejiang province were much higher than in other regions. The contents of orientin and isoorientin in THL from Guizhou, Guangdong, and Guangxi were lower than those from other regions.

\section{CONCLUSION}

In this study, fifteen flavonoids were isolated and identified from THL, four of which were obtained from T. hemsleyanum for the first time. Compounds 2, 4-6, and 9-14 showed significant antioxidant activities. In addition, a new simple and sensitive UPLC-MS/MS method was developed for the separation and quantification of these ten antioxidant compounds, which would provide an evaluable reference for the quality control of T. hemsleyan$u m$. The contents of ten compounds in the THL from different habitats were significantly different. The results suggested that THL could be a new source for functional foods and a potential candidate for antioxidant agents.

\section{Acknowledgments}

This work was supported by the public welfare technology research projects of Zhejiang province (Grant No. LGN18B020001), the Natural Science Foundation of Ningbo city (Grant No. 2016A610235), the Natural Science Foundation of Zhejiang province (Grant No. LQ18C160005), and the Ningbo city science and technology innovation 2025 major research project (Grant No. 2019B10008).

\section{Conflict of interest}

All the authors declare that they have no conflict of interest. 


\section{REFERENCES}

1. $\quad$ Song Y.L., Wu P., Li Y.F., Tong X.X., Zheng Y.F., et al.: Plant Soil 417, 393 (2017).

2. Peng X., Wu H., Chen H.J., Zhang Y.J., Qiu D.: BMC Genom. 20, 687 (2019).

3. Ji W.W., Peng X., Lou T.L., Wang J., Qiu W.Y.: Inflammopharmacology 27, 1297 (2019).

4. Feng Z., Hao W., Lin X., Fan D., Zhou J.: Oncotargets Ther. 7, 947 (2014).

5. Sun Y., Tsao R., Chen F., Li H.Y., Wang J.W., et al.: J. Funct. Foods 30, 179 (2017).

6. Ji T., Ji W.W., Wang J., Chen H.J., Peng X., et al.: J. Ethnopharmacol. 264, 113247 (2021).

7. Ji W.W., Peng X., Lou T.L., Wang J., Qiu W.Y.: Inflammopharmacology 27, 1297 (2019).

8. Shahdadi F., Mirzaei H.O., Garmakhany A.D.: J. Food Sci. Technol. 52, 1814 (2015).

9. Babou L., Hadidi L., Grosso C., Zaidi F., Valentao P., et al.: Eur. Food. Res. Technol. 242, 1447 (2016).

10. Tan L.H., Zhang D., Yu B., Zhao S.P., Cao W.G.: Eur. Food. Res. Technol. 240, 815 (2015).

11. Hu W., Han W., Huang C., Wang M.H.: Environ. Toxicol. Phar. 31, 42 (2011).

12. Tai Z.G., Chen A.Y., Qin B.D., Cai L., Xu Y.Q.: Eur. Food Res. Technol. 239, 501 (2014).

13. Hu W., Yu I., Wang M.H.: Food Chem. Toxicol. 9, 799 (2011).

14. Walia M., Kumar P., Singh B., Agnihotri V.K.: J. Food Sci. Technol. 55, 4337 (2018).

15. Zhou Y., Yuan J.T., Wu J., Han X.D.: Toxicol. Lett. 212, 48 (2012).

16. Li M., Xia Z.M., Li B., Tian Y., Zhang G.J.: Med. Chem. Res. 28, 1557 (2019).

17. Xin Y., Da L.H., Hong Y., Li G.H., Chun L., et al.: Chin. Pharm. J. 4, 264 (2019).

18. Shi S.Y., Cui H.H., Yin Y.Q., Song X.H., Liang H.X., et al.: Chin. Tradit. Herbal Drugs 1, 36 (2019).
19. Yang N.Y., Tao W.W., Duan J.A.: Nat. Prod. Res. 24, 1843 (2010).

20. Dong J.L., Huang C.Q., Huang W., Ma H.R., Liu J.M.: Chin. Tradit. Patent. Med. 41, 1578 (2019).

21. Kazuma K., Noda N., Suzuki M.: Phytochemistry 62, 229 (2003).

22. Yang J., Wang L.L., Zhou X.T., Yuan J.Z., Zhang T.J.: Chin. Tradit. Herbal Drugs 45, 622 (2014).

23. Shao J., Liu Y.L., Li X.R., Xu Q.M., Pei X.J., et al.: Chin. Tradit. Herbal Drugs 47, 1661 (2016).

24. Li Y.P., Zheng C.K., He H.P.: Chin. Med. Mat. 42, 87 (2019).

25. Li S.Y., Wu X.J., She C.W.: Chin. Med. Mat. 34, 604 (2014).

26. Yu L.L., Liu J.C., Chen L.X., Qiu F.: Chin. Tradit. Herbal Drugs 40, 4151 (2016).

27. Yang N.Y., Duan J.A., Li P., Qian S.H.: Chin. Pharm. J. 41, 1621 (2006).

28. Du Y., Wang X.Q., Bao B.Q., Huang H.: Chin. Tradit. Herbal Drugs 47, 2817 (2016).

29. Shu J.C., Zhang R., Zhang W., Pan J.H., Ren X.J., et al.: Chin. Tradit. Patent Med. 34, 1122 (2012).

30. Antonio F., Brigida D., Severina P., Giuseppe C., Piera U.: Biochem. Syst. Ecol. 36, 691(2008).

31. Margareth B.C., Paulo C.V., Joao B.F.: J. Ethnopharmacol. 115, 320 (2008).

32. Liu Z.W., Que Z.L., Ye Z.W., Zhang X.Q., Wang H., et al.: Chin. J. Nat. Med. 6, 415 (2008).

33. Verma A.K., Pratap R.: Nat. Prod. Rep. 27, 571 (2010).

34. Panche A.N., Diwan A.D., Chandra S.R.: J. Nutr. Sci. 5, 1 (2016).

35. Hussein M. Ali., Isra H. Ali.: Med. Chem. Res. 28, 2262 (2019).

36. Zheng W.F., Zhao Y.X., Zheng X., Liu Y.B., Pan S.Y.: Appl. Microbiol. Biotechnol. 89, 157 (2011).

37. Roginsky V., Lissi E.A.: Food Chem. 92, 235 (2005).

38. Djeradi H., Rahmouni A., Cheriti A.: J. Mol. Model. 20, 2476 (2014). 
